\title{
EFEITO DO EXERCÍCIO FÍSICO SOBRE A SAÚDE E SOBRECARGA DE MÃES DE CRIANÇAS E ADOLESCENTES COM PARALISIA CEREBRAL
}

\author{
EFFECT OF EXERCISE ON HEALTH AND BURDEN OF MOTHERS OF CHILDREN \\ AND ADOLESCENTS WITH CEREBRAL PALSY
}

EFECTO DEL EJERCICIO SOBRE LA SALUD Y SOBRECARGA DE LAS MADRES DE NIÑOS

Y ADOLESCENTES CON PARÁLISIS CEREBRAL

\author{
Moisés Rosa Batista \\ (Educador físico) \\ Jaqueline Pontes Batista \\ (Educadora física) \\ Jussara Caetano Furtado \\ (Educadora física) \\ Luiz Duarte de Ulhôa Rocha Junior ${ }^{1}$ \\ (Educador físico) \\ Eduardo Henrique Tavares \\ (Educador físico) \\ Hygor Nunes Araújo \\ (Educador físico) \\ Rogério de Melo Costa Pinto \\ (Engenheiro Agrônomo) \\ Nívea de Macedo Oliveira Morales \\ (Médica Neuropediatra)
}

1. Universidade Federal de Uberlândia, Uberlândia, MG, Brasil.

\section{Correspondência:}

Nívea de Macedo Oliveira Morales Avenida Pará, 1720, Bairro Umuarama, Uberlândia, MG, Brasil. 38400-902. nivea.neuroped@gmail.com

\section{RESUMO}

Introdução: A paralisia cerebral (PC) é considerada a incapacidade física mais comum na infância. Essa doença afeta profundamente a saúde e o bem-estar dos indivíduos acometidos e também pode influenciar múltiplos aspectos da vida de seus cuidadores, especialmente as mães. Objetivo: Avaliar o efeito de um programa de exercício resistido sobre a qualidade de vida relacionada à saúde (QVRS) de mães de crianças e adolescentes com PC. Método: Vinte e duas mães sedentárias cuidadoras de crianças e adolescentes com PC, aptas à prática de exercício resistido, participaram de um programa de exercício resistido de intensidade moderada, em 2 sessões semanais durante 12 semanas. Todos os participantes responderam ao questionário de QVRS, Short Form Questionnaire (SF-36), à escala de sobrecarga Caregiver Burden Scale (CBS) e ao Inventário de Depressão de Beck (BDI) antes e após o programa de intervenção. Os escores dos questionários pré e pós-intervenção foram comparados pelo teste de Wilcoxon e a magnitude das diferenças foi medida pelo tamanho do efeito. Resultados: A mediana de idade das mães foi de 41 anos e variou de 18 a 58 anos. A mediana da idade das crianças/adolescentes foi de 14 anos, variando 3 a 21 anos. Após a intervenção foram encontrados aumentos significativos nos escores do SF-36 $(p<0,05)$, exceto nos domínios aspectos físicos e aspectos emocionais, que já obtiveram pontuação máxima pré-intervenção. Os escores do CBS e do BDI tiveram redução significativa pós-intervenção $(p<0,05)$. Conclusão: A prática regular de exercícios resistidos tem impacto positivo sobre a QVRS, a percepção de sobrecarga e a intensidade de sintomas depressivos de mães cuidadoras de crianças e adolescentes com PC.

Descritores: treinamento de resistência; depressão; cuidadores.

\section{ABSTRACT}

Introduction: Cerebral palsy (CP) is considered the most common physical disability in childhood. This disease profoundly affects the health and well-being of affected individuals and can influence multiple aspects of the life of their caregivers, especially mothers. Objective: To evaluate the effect of a resistance training program on health-related quality of life (HRQoL) of mothers of children and adolescents with CP. Methods: Twenty-four sedentary mothers caregivers of children and adolescents with CP, able to practice resistance training, participated in a resistance training program of moderate intensity of 2 sessions per week for 12 weeks. All participants answered to HRQoL questionnaire, SF-36, Caregiver Burden Scale (CBS) and Beck Depression Inventory (BDI) before and after the intervention program. The scores of the questionnaires before and after the intervention were compared using the Wilcoxon test and the magnitude of the differences was measured by effect size. Results: The median age of the mothers was 41 years, ranging from 18 to 58 years. The median age of children and adolescents was 14 years, ranging from 3 to 21 years. The SF-36 scores were significantly higher after the intervention ( $p<0.05)$, except in the domains physical aspects and emotional aspects, which already scored the highest value before the intervention. $C B S$ and $B D I$ scores were significantly reduced after intervention $(p<0.05)$. Conclusion: The regular practice of resistance training has a positive impact on HRQOL, burden perception and intensity of depressive symptoms of mothers caregivers of children and adolescents with CP.

Keywords: resistance training; depression; caregivers.

\section{RESUMEN}

Introducción: La parálisis cerebral (PC) es considerada la discapacidad física más común en la infancia. Esta enfermedad afecta profundamente la salud y el bienestar de los individuos afectados y también puede influir en muchos aspectos de la vida de sus cuidadores, especialmente las madres. Objetivo: Evaluar el efecto de un programa de ejercicios de resistencia en la calidad de vida relacionada con la salud (CVRS) de las madres de niños y adolescentes con parálisis cerebral. Método: Veinte y dos madres sedentarias cuidadoras de niños y adolescentes con PC, aptas para la práctica de ejercicios de resistencia, participaron en un programa de ejercicios de resistencia de intensidad 
moderada, de 2 sesiones por semana durante 12 semanas. Todos los participantes respondieron el cuestionario CVRS, el Short Form Questionnaire (SF-36), la escala de sobrecarga del cuidador Caregiver Burden Scale (CBS) y el Inventario de Depresión de Beck (BDI) antes y después de la intervención. Las puntuaciones de los cuestionarios antes y después de la intervención se compararon mediante la prueba de Wilcoxon y la magnitud de las diferencias fue medida por el tamaño del efecto. Resultados: La edad mediana de las madres fue de 41 años, con un rango de 18 a 58 años. La mediana de edad de los niños/adolescentes fue de 14 años, con rango 3 a 21 años. Después de la intervención se encontró un aumento significativo en los resultados de SF-36 $(p<0,05)$, excepto en los dominios aspectos físicos y aspectos emocionales, que han obtenido la máxima puntuación antes de la intervención. Las puntuaciones de la CBS y la BDI tuvieron una reducción significativa después de la intervención $(p<0,05)$. Conclusión: La práctica regular de ejercicios de resistencia tiene un impacto positivo en la CVRS, la percepción de la sobrecarga y la intensidad de los sintomas depresivos de las madres cuidadoras de niños y adolescentes con PC.

Descriptores: entrenamiento de resistencia; depresión; cuidadores.

\section{INTRODUÇÃO}

A paralisia cerebral (PC) é uma importante causa de incapacidade na infância com prevalência aproximada de 2/1000 nascidos vivos'. Decorre de uma lesão no cérebro em desenvolvimento e é caracterizada por desordens do movimento e da postura que ocasionam limitação motora, de caráter não progressivo². Embora as manifestações neurológicas sejam observadas precocemente, a PC é considerada uma condição crônica que gera limitações funcionais a longo prazo ${ }^{3}$ e pode interferir em múltiplos aspectos da vida dos pacientes e de seus familiares ${ }^{4-8}$. O difícil processo de cuidar de uma criança ou adolescente com PC pode ocasionar cansaço, isolamento, sobrecarga e estresse nos cuidadores ${ }^{7}$. As mães, que em geral são as cuidadoras principais, apresentam mais sintomas depressivos e elevados níveis de sobrecarga, além de um significativo impacto negativo em sua qualidade de vida (QV) $4,6,8$.

A QV pode ser definida como um bem-estar subjetivo, uma satisfação ou felicidade pessoal com a vida nos domínios que o indivíduo considera importante, como o físico, psicológico, social, econômico e espiritual ${ }^{9}$. A qualidade de vida relacionada à saúde (QVRS) refere-se à satisfação e bem-estar do indivíduo em relação ao estado de saúde ${ }^{10}$. Embora os serviços de saúde direcionem seus recursos na promoção da saúde e da QVRS dos pacientes, poucas intervenções são oferecidas aos familiares e aos cuidadores. A negligência com sua própria saúde e a inatividade física são bastante frequentes entre os cuidadores de pacientes com doenças crônicas"1".

Alguns estudos demonstraram benefício do exercício físico para cuidadores de idosos ou de pessoas com Alzheimer quanto à QV ${ }^{12,13} \mathrm{e}$ aos aspectos físicos e mentais da saúde ${ }^{11-16}$. De fato, há considerável e crescente evidência de que o exercício físico desempenha um importante papel na promoção da saúde, na percepção de QV e nos sintomas depressivos (11,13,17-20. $^{\circ}$ treinamento resistido regular tem sido proposto como uma ferramenta efetiva na prevenção de perdas de massa muscular e na redução de eventos cardiovasculares, além de proporcionar melhoras no desempenho físico, na capacidade funcional e no bem estar gera ${ }^{21}$. Contudo, há pouca informação na literatura sobre o efeito do exercício resistido na QVRS ou na percepção de sobrecarga de cuidadores de pacientes com doenças crônicas ${ }^{16}$ e nenhuma pesquisa incluiu mães de crianças com doenças neurológicas incapacitantes, como a PC. Como esse grupo de mães apresenta particularidades em função da vulnerabilidade de estar exposto a uma constante sobrecarga física e mental diária4, é importante considerar a percepção subjetiva do próprio indivíduo e para o planejamento de futuras estratégias de intervenção. O presente estudo tem como objetivo avaliar o efeito do exercício resistido na QVRS, intensidade de sintomas depressivos e níveis de sobrecarga de mães cuidadoras de crianças e adolescentes com PC.

\section{MATERIAIS E MÉTODOS}

O estudo intervencional foi aprovado pelo Comitê de Ética em Pesquisa da Universidade Federal de Uberlândia (protocolo 172/10) e conduzido entre o período de janeiro de 2011 e dezembro de 2011.

Foram convidados a participar as mães cuidadoras de crianças e adolescentes com diagnóstico de PC com idade de até 21 anos atendidas no Hospital de Clínicas da Universidade Federal de Uberlândia (HC/UFU). Segundo os critérios de inclusão: idade entre 20 e 59 anos; sedentária, ou seja, não realizar atividade física regular de forma programada e sistematizada por pelo menos duas vezes na semana; consentimento e assinatura do Termo de Consentimento Livre e Esclarecido (TCLE) após esclarecimento sobre o protocolo da pesquisa. Os critérios de exclusão foram: duas faltas consecutivas ou desistência de participar do estudo; cuidar de mais de um indivíduo com doença crônica; apresentar contraindicação à prática do exercício anaeróbio; durante o estudo iniciar um novo tratamento médico ou terapêutico que potencialmente influencie as variáveis analisadas ou que contraindiquem a manutenção da atividade física proposta.

Todas as mães participantes responderam uma entrevista estruturada, para a obtenção de dados demográficos sobre as mães (idade, escolaridade, situação conjugal, renda familiar, vínculo empregatício) e clínicos (uso de medicações, doença crônica, presença de outro membro da família com doença crônica e se o mesmo está sob os cuidados do participante da pesquisa). Foram obtidas do prontuário médico do Hospital de Clínicas da Universidade Federal de Uberlândia HC/UFU as informações clínicas e demográficas da criança e adolescentes com PC.

Todas as mães responderam ao Medical Outcomes Survey Short-Form 36 (SF-36) 22 para avaliar a sua própria QVRS, ao Inventário de Depressão de Beck (BDI) $)^{23}$ para avaliar os sintomas depressivos e o Caregiver Burden Scale (CBS) ${ }^{24}$ para medir os níveis de sobrecarga. Esses questionários foram desenvolvidos originalmente na língua inglesa e utilizada a versão brasileira, previamente traduzida, adaptada e validada (Tabela 1). Foram feitas avaliações antropométricas (peso, altura, percentual de gordura e IMC) e de valências físicas (teste de resistência do abdômen, teste de resistência dos braços e teste de flexibilidade). Todos os questionários e avaliações foram repetidos após a intervenção.

As sessões de treinamento resistido (musculação) foram realizadas na Academia de Musculação da Faculdade de Educação Física da Universidade Federal de Uberlândia (FAEFI/UFU). Todos os voluntários foram submetidos à avaliação médica antes do início dos exercícios por um médico fisiatra. As voluntárias sem contraindicação médica para a prática dos exercícios foram submetidas ao treinamento com pesos (exercício resistido). A carga para o treinamento foi definida pelo teste de ensaio e erro ${ }^{25}$. Os exercícios escolhidos para a 
Tabela 1. Instrumentos de avaliação utilizados na pesquisa.

\begin{tabular}{|c|c|c|c|}
\hline Instrumento & $\begin{array}{l}\mathrm{n}^{\circ} \text { de } \\
\text { itens }\end{array}$ & $\begin{array}{l}\text { Descrição do instrumento e } \\
\text { modo de aplicação }\end{array}$ & $\begin{array}{l}\text { Amplitude dos } \\
\text { escores }\end{array}$ \\
\hline $\begin{array}{l}\text { Medical } \\
\text { Outcomes Survey } \\
\text { Short-Form } 36^{21}\end{array}$ & 36 & $\begin{array}{l}\text { Questionário genérico de } \\
\text { QVRS. Contém oito domínios } \\
\text { (capacidade física, aspectos } \\
\text { físicos, dor, estado geral de saú- } \\
\text { de, vitalidade, aspectos sociais, } \\
\text { aspectos emocionais e saúde } \\
\text { mental) e dois componentes } \\
\text { sumários (físico e mental). Apli- } \\
\text { cado por meio de entrevista. }\end{array}$ & $\begin{array}{c}\text { 0-100 (quanto maior } \\
\text { o escore, melhor a } \\
\text { QVRS) }\end{array}$ \\
\hline $\begin{array}{l}\text { Caregiver Burden } \\
\text { Scale }(\mathrm{CBS})^{23}\end{array}$ & 22 & $\begin{array}{l}\text { Avalia o impacto subjetivo do } \\
\text { ato de cuidar dos cuidadores } \\
\text { de pacientes com doenças } \\
\text { crônicas. As questões são } \\
\text { divididas em } 5 \text { domínios: } \\
\text { tensão geral, isolamento, decep- } \\
\text { ção, envolvimento emocional e } \\
\text { ambiente. Aplicado por meio } \\
\text { de entrevista. }\end{array}$ & $\begin{array}{l}\text { 22-88 (quanto maior } \\
\text { o escore, maior o } \\
\text { grau de dificuldade } \\
\text { percebido pelo } \\
\text { cuidador) }\end{array}$ \\
\hline $\begin{array}{l}\text { Inventário de } \\
\text { Depressão de } \\
\text { Beck }(B D I)^{22}\end{array}$ & 21 & $\begin{array}{c}\text { Avalia a intensidade de } \\
\text { sintomas depressivos. } \\
\text { Auto-aplicado. }\end{array}$ & $\begin{array}{l}\text { 0-63 (quanto maior } \\
\text { escore, maior a in- } \\
\text { tensidade de sinto- } \\
\text { mas depressivos) }\end{array}$ \\
\hline
\end{tabular}

realização do trabalho visaram os grupos musculares principais (peitoral, ombros, costas, quadris, pernas, tronco e braços), assim como exercícios que isolam grupos musculares funcionalmente importantes (abdominais e extensores lombares: supino reto (peitoral), puxador vertical frente com barra triângulo (grande dorsal), leg press (quadríceps), mesa flexora (ísquios-tibiais), abdominal deitado (abdome), rosca bíceps (bíceps), tríceps com polia alta (tríceps), remada alta (deltóide) e extensão do tronco no banco romano (para vertebrais). Durante 12 semanas, com frequência de duas vezes na semana, em dias alternados e com duração de 60 minutos cada sessão, as voluntárias realizaram três séries de quinze repetições máximas em cada um dos exercícios propostos, sendo que, cada vez que a voluntária conseguia realizar mais que quinze repetições de forma confortável a carga era aumentada. O intervalo entre as séries foram de um minuto ${ }^{21}$. Os alongamentos foram feitos de forma ativa, ou seja, pela própria voluntária após o treinamento.

\section{Análise estatística}

A estatística descritiva foi utilizada para a caracterização demográfica e clínica dos participantes e para determinar os escores do SF-36, CBS e do BDI. Os dados não tiveram distribuição normal segundo o teste Lilliefors, sendo utilizados testes não paramétricos.

O coeficiente de alpha-Cronbach foi utilizado para verificar a confiabilidade da consistência interna obtida nos domínios do SF-36. Os escores do SF-36, CBS e do BDI foram comparados pelo teste de Wilcoxon antes e depois da intervenção com exercício resistido. O tamanho do efeito foi calculado como a razão entre a diferença das medianas e o desvio interquartílico da primeira avaliação para medir a magnitude das diferenças significativas na comparação entre a primeira e segunda avaliação. Valores maiores ou iguais a 0,8 são considerados grandes, de 0,4 a 0,79 moderados e abaixo de 0,4 pequenos. Os dados obtidos pela antropometria e testes de resistência muscular localizada foram comparados pelo teste de Wilcoxon antes e depois da intervenção com exercício resistido. Os dados foram analisados por meio do software estatístico Statistical Package for Social Science versão 18.0 (SPSS Inc., Chicago, IL, EUA). O nível de significância adotado foi de $\mathrm{p}<0,05$.

\section{RESULTADOS}

O banco de dados consultado do HC/UFU forneceu contato telefônico de 700 mães de crianças e adolescentes com PC que foram convidadas a participar do estudo. Deste total apenas 88 compareceram à reunião para maior esclarecimento sobre a pesquisa, porém 44 não concordaram em participar, a maioria por relatar não ter condições de deixar seu filho com PC para comparecer às sessões de treinamento. Das 44 voluntárias que concordaram em participar, 22 mães foram excluídas: 20 por desistência logo nas primeiras semanas; uma por apresentar contraindicação cardiológica e outra por iniciar tratamento medicamentoso com antidepressivo durante a intervenção. Portanto, participaram do estudo 22 mães. (Tabela 2)

A maioria das crianças/adolescentes com PC eram do sexo masculino $(68,18 \%)$ com mediana de idade de 14 anos. Com relação ao tipo de PC 22,72\% tinham a forma tetraparética e 36,36\% com grave comprometimento motor (nível 5 pelo Gross Motor Classification System). Onze pacientes (50\%) apresentavam algum tipo de comorbidade relacionada à PC e 14 (63,63\%) já haviam sido submetidos a procedimento cirúrgico.

$\mathrm{Na}$ avaliação da confiabilidade do SF-36, os valores encontrados para o alpha-Cronbach variaram de 0,74 a 0,90 pré-intervenção e de 0,41 a 0,91 pós-intervenção. Valores abaixo de 0,50 foram obtidos apenas no domínio vitalidade pós- intervenção.

Quanto à avaliação da QVRS, houve diferença significativa em 5 domínios do SF-36 e no componente físico, com maiores escores pós-intervenção $(p<0,05)$ (Tabela 3)

Os escores do BDI e do CBS foram significativamente menores no período pós-intervenção $(p<0,05)$ (Tabela 4).

Os resultados das comparações entre os dados antropométricos antes e após a intervenção mostraram diferenças significativas com redução do percentual de gordura (36,41 vs. 35,14 $p=0,02)$ e massa gorda (22,64 vs. $22,54 \mathrm{p}=0,04)$; aumento de massa magra (42,06 vs. 43,24 $p=0,03)$, flexibilidade $(23,50$ vs. $24,50 p=0,01)$, resistência de braços $(13,50$ vs. $17,50 p=0,04)$ e resistência abdominal $(24,50$ vs. $30,00 p=0,03)$.

\section{DISCUSSÃO}

Este trabalho representa o primeiro estudo a avaliar e detectar os benefícios do exercício resistido na qualidade de vida relacionada à saúde, intensidade de sintomas depressivos e níveis de sobrecarga de mães cuidadoras de crianças e adolescentes com paralisia cerebral.

A utilização de um instrumento genérico permitiu observar a repercussão positiva do treinamento resistido tanto na dimensão física

Tabela 2. Características demográficas e clínicas das mães participantes do estudo.

\begin{tabular}{c|c}
\hline Características & Valor $(\mathbf{n = 2 2})$ \\
\hline Idade & 41,00 \\
\hline Mediana & $30,75-47,25$ \\
\hline Percentil 25 - 75 & $15(68,18 \%)$ \\
\hline Atividade remunerada $\mathbf{n}$ (\%) & 2 \\
\hline Renda (em salários mínimos) & $1,50-3,62$ \\
\hline Mediana & 10 \\
\hline Percentil 25 - 75 & $6,75-11,00$ \\
\hline Escolaridade (anos de estudo) & $15(68,18 \%)$ \\
\hline Mediana & $6(27,27 \%)$ \\
\hline Percentil 25 - 75 & $3(13,64 \%)$ \\
\hline União marital estável $\mathbf{n}(\%)$ & $2(9,09 \%)$ \\
\hline Sim & $1(4,55 \%)$ \\
\hline Doenças crônicas $\mathbf{n}(\%)$ & \\
\hline Sim & \\
\hline - Hipotireoidismo & \\
\hline - Hipertensão arterial & \\
\hline - Doença de Cronh & \\
\hline
\end{tabular}


Tabela 3. Escores do SF-36 obtidos pelas mães participantes do estudo.

\begin{tabular}{|c|c|c|c|c|}
\hline \multirow[b]{2}{*}{$\begin{array}{l}\text { Domínios e } \\
\text { componentes }\end{array}$} & Pré-intervenção & Pós-intervenção & \multirow[b]{2}{*}{ p valor* } & \multirow{2}{*}{$\begin{array}{c}\text { Tamanho do } \\
\text { efeito }\end{array}$} \\
\hline & \multicolumn{2}{|c|}{$\begin{array}{c}\text { Mediana } \\
\text { (Percentil 25 - 75) }\end{array}$} & & \\
\hline $\begin{array}{l}\text { Capacidade } \\
\text { funcional }\end{array}$ & $\begin{array}{c}85,00 \\
(70,00-95,00)\end{array}$ & $\begin{array}{c}90,00 \\
(85,00-95,00)\end{array}$ & 0,01 & 0,20 \\
\hline Aspectos físicos & $\begin{array}{c}100,00 \\
(43,75-100,00)\end{array}$ & $\begin{array}{c}100,00 \\
(93,75-100,00)\end{array}$ & 0,38 & - \\
\hline Dor & $\begin{array}{c}52,00 \\
(51,00-72,00)\end{array}$ & $\begin{array}{c}78,00 \\
(62,00-100,00)\end{array}$ & 0,00 & 1,24 \\
\hline $\begin{array}{l}\text { Estado geral de } \\
\text { saúde }\end{array}$ & $\begin{array}{c}72,00 \\
(57,75-83,25) \\
\end{array}$ & $\begin{array}{c}86,00 \\
(70,75-92,00) \\
\end{array}$ & 0,01 & 0,55 \\
\hline Vitalidade & $\begin{array}{c}55,00 \\
(37,50-71,25) \\
\end{array}$ & $\begin{array}{c}77,50 \\
(62,50-85,00) \\
\end{array}$ & 0,00 & 0,66 \\
\hline Aspectos sociais & $\begin{array}{c}75,00 \\
(43,75-100,00)\end{array}$ & $\begin{array}{c}100,00 \\
(84,37-100,00) \\
\end{array}$ & 0,01 & 0,44 \\
\hline $\begin{array}{c}\text { Aspectos } \\
\text { emocionais }\end{array}$ & $\begin{array}{c}100,00 \\
(66,67-100,00) \\
\end{array}$ & $\begin{array}{c}100,00 \\
(66,67-100,00) \\
\end{array}$ & 0,67 & - \\
\hline Saúde mental & $\begin{array}{c}74,00 \\
(52,00-84,00)\end{array}$ & $\begin{array}{c}76,00 \\
(64,00-88,00)\end{array}$ & 0,04 & 0,06 \\
\hline $\begin{array}{l}\text { Componente } \\
\text { físico }\end{array}$ & $\begin{array}{c}50,52 \\
(44,91-53,45) \\
\end{array}$ & $\begin{array}{c}55,06 \\
(52,68-58,71) \\
\end{array}$ & 0,01 & 0,53 \\
\hline $\begin{array}{c}\text { Componente } \\
\text { mental }\end{array}$ & $\begin{array}{c}50,05 \\
(42,78-52,72) \\
\end{array}$ & $\begin{array}{c}51,53 \\
(43,99-57,72) \\
\end{array}$ & 0,12 & - \\
\hline
\end{tabular}

Tabela 4. Escores do inventário de depressão (BDI) e da escala de sobrecarga (CBS) obtido pelas mães participantes do estudo.

\begin{tabular}{|c|c|c|c|c|}
\hline Instrumento & $\begin{array}{c}\text { Pré-Intervenção } \\
\text { Mediana } \\
\text { (Percentil } 25-75)\end{array}$ & $\begin{array}{c}\text { Pós-Intervenção } \\
\text { Mediana } \\
\text { (Percentil } 25 \text { - 75) }\end{array}$ & p valor* & $\begin{array}{l}\text { Tamanho } \\
\text { do efeito }\end{array}$ \\
\hline \multicolumn{3}{|l|}{ BDI } & \multirow[b]{2}{*}{0,04} & \multirow[b]{2}{*}{$-0,67$} \\
\hline $\begin{array}{c}\text { Mediana } \\
\text { Percentil } 25-75\end{array}$ & $\begin{array}{c}9,50 \\
5,00-13,25\end{array}$ & $\begin{array}{c}4,00 \\
2,00-10,25\end{array}$ & & \\
\hline \multicolumn{3}{|l|}{ Escore total - CBS } & \multirow{3}{*}{0,02} & \multirow{3}{*}{$-0,82$} \\
\hline Mediana & 37,50 & 29,50 & & \\
\hline Percentil 25 - 75 & $31,50-41,25$ & $25,75-39,25$ & & \\
\hline \multicolumn{3}{|l|}{ Tensão geral - CBS } & \multirow{3}{*}{0,02} & \multirow{3}{*}{$-0,36$} \\
\hline Mediana & 1,75 & 1,29 & & \\
\hline Percentil 25 - 75 & $1,41-2,23$ & $1,13-1,75$ & & \\
\hline \multicolumn{3}{|l|}{ Isolamento - CBS } & \multirow{3}{*}{0,03} & \multirow{3}{*}{$-1,23$} \\
\hline Mediana & 1,70 & 1,16 & & \\
\hline Percentil $25-75$ & $1,43-1,87$ & $1,00-1,67$ & & \\
\hline \multicolumn{3}{|l|}{ Decepção - CBS } & \multirow{3}{*}{0,04} & \multirow{3}{*}{$-0,25$} \\
\hline Mediana & 1,60 & 1,40 & & \\
\hline Percentil $25-75$ & $1,40-2,20$ & $1,00-1,80$ & & \\
\hline \multicolumn{3}{|l|}{$\begin{array}{c}\text { Envolvimento emo- } \\
\text { cional - CBS }\end{array}$} & \multirow{3}{*}{0,10} & \multirow{3}{*}{-} \\
\hline Mediana & 1,00 & 1,00 & & \\
\hline Percentil $25-75$ & $1,00-1,42$ & $1,00-1,00$ & & \\
\hline \multicolumn{3}{|l|}{ Ambiente - CBS } & \multirow{3}{*}{0,56} & \multirow{3}{*}{-} \\
\hline Mediana & 1,67 & 1,67 & & \\
\hline Percentil $25-75$ & $1,00-2,00$ & $1,00-2,00$ & & \\
\hline
\end{tabular}

quanto mental da QVRS dessas mães. As melhoras foram mais evidentes nos domínios físicos do que nos mentais, de acordo com os sumários do componente físico do SF-36. Resultados positivos na percepção de saúde física dessas mães ocorreram principalmente nos domínios que avaliam a capacidade física, dor e estado geral de saúde. Deve-se destacar que o domínio dor da QVRS foi o único que apresentou tamanho de efeito elevado com a intervenção. Como esse domínio avalia o quanto a dor corporal influencia nas atividades cotidianas, as melhoras obtidas podem favorecer e facilitar as tarefas desempenhadas por essas mães cuidadoras.

Embora os benefícios do exercício físico na QV tenham sido relatados em outros grupos de indivíduos com doenças crônicas e saudáveis ${ }^{14,17,18}$, a utilização do mesmo na promoção de saúde para os cuidadores de pacientes com doenças neurológicas incapacitantes ainda não está amplamente disponível. Isso foi confirmado de modo indireto no presente estudo ao se verificar que todas as mães convidadas a participar eram sedentárias. A atenção ao cuidador e às mães de crianças e adolescentes com PC ainda é limitada.

Além dos domínios físicos da QVRS, as variáveis antropométricas aqui avaliadas antes e após a intervenção também mostraram resultados positivos, ou seja, houve uma redução no percentual de gordura corporal e na massa gorda, enquanto aumentos significativos foram observados na flexibilidade, massa magra e nos testes de resistência abdominal e resistência dos braços. Esses resultados devem proporcionar uma melhora na força muscular, mobilidade e funcionalidade dessas mães, o que pode facilitar as atividades do cotidiano.

Apesar dos resultados mais expressivos do presente estudo terem ocorrido nos domínios físicos, melhoras também foram identificadas na dimensão mental da QVRS dessas mães, pelos domínios vitalidade, aspectos sociais e saúde mental. A intensidade de sintomas depressivos avaliados por meio do BDI após o período de treinamento também mostrou melhora significativa e de moderada magnitude.

Resultados semelhantes são relatados em estudos prévios com populações diversas, incluindo cuidadores, e reforçam o benefício do exercício físico na saúde mental em geral e no tratamento e prevenção da depressão 13-16,20,26,27. Após a intervenção observou-se também redução dos níveis de sobrecarga segundo o CBS nos domínios tensão geral, isolamento e decepção. Deve-se destacar que a melhora obtida no escore total de sobrecarga e na percepção de isolamento foram de elevada magnitude. Este achado confirma o benefício da atividade física na percepção de sobrecarga e na socialização das mães que assumem o papel de cuidadores.

Não foram encontrados na literatura trabalhos de intervenção com cuidadores que utilizaram como forma de avaliação dos níveis de sobrecarga o mesmo instrumento do presente estudo. Porém, a melhora na percepção subjetiva de sobrecarga de cuidadores de pacientes com demência foi detectada após treino aeróbico ${ }^{14}$ e atividade aeróbia combinada com treinamento resistido ${ }^{16}$.

Um estudo que avaliou a QVRS de cuidadores de idosos por meio de intervenção com exercício resistido e outras modalidades como thai chi, yoga e treinamento em circuito, mostrou resultados semelhantes quanto às melhoras físicas na QVRS, além de melhoras nos sintomas depressivos, equilíbrio, força em membros inferiores e resistência ao caminhar. Esses cuidadores relataram também, que o programa foi agradável, lhes proporcionando tempo para cuidar de suas próprias necessidades e que Ihes permitiu uma ruptura completa do papel de cuidador $^{13}$. O exercício físico regular também tem sido proposto e apresenta eficácia na melhora de sintomas depressivos, ansiedade, saúde física e geral, e na percepção de sobrecarga em cuidadores de idosos e cuidadores de pessoas com Alzheimer ${ }^{11-16}$.

O treinamento resistido foi escolhido no presente estudo porque previne perdas de massa muscular, além de melhorar o desempenho físico, a funcionalidade e o bem-estar ${ }^{19}$. A força muscular e a resistência muscular promovidas pelo treinamento resistido exercem um impacto direto sobre as atividades da vida diária das pessoas, portanto, o aprimoramento dessas valências permite aos indivíduos a realização das tarefas cotidianas com menos estresse fisiológico e ajuda na manutenção da independência funcional ao longo da vida ${ }^{21}$. Esses benefícios são desejáveis na vida dos cuidadores de pacientes com doenças crônicas.

Apesar dos efeitos positivos do exercício físico em geral, a condição de cuidador é reconhecidamente um fator limitante para a prática regular de atividade física, assim como para o tempo gasto em atividades de lazer, o que pode refletir de forma negativa na saúde dos cuidadores ${ }^{14}$. 
A PC, como doença incapacitante da infância, gera grandes repercussões na realização de tarefas simples e corriqueiras, o que afeta a rotina das próprias crianças e de seus familiares ${ }^{5,6}$. As mães, que em geral são as cuidadoras principais, apresentam uma sobrecarga emocional, física e financeira, além de restrições nas atividades sociais e de lazer, com consequente prejuízo na QVRS ${ }^{4-6,8,16}$. Estratégias de intervenção para minimizar esses efeitos são essenciais e os resultados do presente estudo reforçam o papel do exercício físico em vários aspectos na vida dessas mães cuidadoras.

Algumas questões metodológicas deste estudo também devem ser discutidas. Embora o SF-36 seja o instrumento mais utilizado para avaliar a QVRS de mães ${ }^{28}$, deve-se considerar a possibilidade deste questionário não ter sido capaz de detectar algumas particularidades desse grupo. Como exemplo, pode-se verificar que nos domínios aspectos físicos e aspectos emocionais os escores já apresentavam valor máximo antes da intervenção (efeito teto), sendo o instrumento incapaz de medir alteração além desse valor. O pequeno número de participantes desta pesquisa, também constitui uma limitação na generalização dos resultados. Deve-se considerar também a pequena taxa de adesão ao estudo e que a maior dificuldade relatada pelas mães para participar foi atribuída à necessidade de cuidar do filho com PC. Deve-se considerar que a sobrecarga imposta pela condição de ser cuidadora impossibilitou grande parte das mães de participar de um programa que as beneficiaria. Partindo do pressuposto desta dificuldade de adesão às intervenções por falta de tempo, alguns autores têm sugerido intervenções domiciliares ${ }^{16,20}$. A eficácia das intervenções na prática clínica deve procurar satisfazer as necessidades, desejos e interesses dos participantes, assim como minimizar o problema com o transporte até o local e a disponibilidade de um cuidador substituto ${ }^{29}$.

Deve-se ainda ressaltar que mesmo após a finalização da intervenção, as mães foram orientadas e estimuladas a manter a prática de exercício físico e não retornar a condição sedentária de antes.
Os resultados desta pesquisa implicam na necessidade de um olhar mais atento para essas mães, visto que, a maioria dos serviços de saúde, projetos acadêmicos e pesquisas são direcionados para crianças ou adolescentes acometidos pela doença. As intervenções devem ir além dos diagnósticos preestabelecidos e atingir os familiares, especialmente as mães e os cuidadores. A atividade aqui proposta e também outras formas de intervenção por meio de atividade física podem ser planejadas para a melhoria do bem estar dessas mães com um custo não elevado e disponibilizada pelas próprias instituições que atendem os pacientes, o que facilitaria a adesão e atingiria um maior número de mães.

\section{CONCLUSÃO}

O exercício físico resistido ocasiona um impacto positivo na qualidade de vida relacionada à saúde, tanto nos domínios físicos como mentais, nos sintomas depressivos e níveis de sobrecarga de mães cuidadoras de crianças e adolescentes com paralisia cerebral. A prática regular de musculação promoveu uma redução do percentual de gordura e da massa gorda e um aumento na massa magra, na flexibilidade e na resistência dos braços e do abdômen para essas mães cuidadoras.

\section{AGRADECIMENTOS}

Aos órgãos financiadores da pesquisa CAPES, CNPq e FAPEMIG. Ao Hospital de Clínicas da Universidade Federal de Uberlândia por fornecer o banco de dados dos pacientes e a Faculdade de Educação Física da Universidade Federal de Uberlândia onde foram realizadas as intervenções. Ao médico fisiatra Fausto Gonzaga de Freitas Filho pela avaliação clínica dos participantes da pesquisa.

Todos os autores declararam não haver qualquer potencial conflito de interesses referente a este artigo.

CONTRIBUIÇÕES DOS AUTORES: Cada autor contribuiu individual e significativamente para o desenvolvimento do manuscrito. MRB (0000-0001-7133-7012)* e NMOM (0000-0002-6111-9318)* foram os principais contribuintes na redação do manuscrito. JPB (0000-0003-2712-935X)*, JCF(0000-0002-3372-6516)*, EHT (0000-0001-5310-1321)* e HNA (0000-0003-1553-2635)*, realizaram o treinamento, acompanharam as voluntárias e reuniram dados clínicos. LDURJ (0000-00023255-007X)*e RMCP (0000-0002-3397-5803)* avaliaram os dados da análise estatística. MRB e NMOM realizaram a pesquisa bibliográfica, a revisão do manuscrito e contribuíram com o conceito intelectual do estudo. *Número ORCID (Open Researcher and Contributor ID).

\section{REFERÊNCIAS}

1. Himmelmann K, Hagberg G, Uvebrant P. The changing panorama of cerebral palsy in Sweden. X. Prevalence and origin in the birth-year period 1999-2002. Acta Paediatr. 2010;99(9):1337-43.

2. Bax M, Goldstein M, Rosenbaum P, Leviton A, Paneth N, Dan B, et al. Proposed definition and classification of cerebral palsy, April 2005. Dev Med Child Neurol. 2005;47(8):571-6.

3. Racine $E$, Larivière-Bastien D, Bell E, Majnemer A, Shevell M. Respect for autonomy in the healthcare context: observations from a qualitative study of young adults with cerebral palsy. Child Care Health Dev. 2013;39(6):873-9.

4. Eker L, Tüzün EH. An evaluation of quality of life of mothers of children with cerebral palsy. Disabil Rehabil. 2004;26(23):1354-9.

5. Ones K, Yilmaz E, Cetinkaya B, Caglar N. Assessment of the quality of life of mothers of children with cerebral palsy (primary caregivers). Neurorehabil Neural Repair. 2005;19(3):232-7.

6. Byrne MB, Hurley DA, Daly L, Cunningham CG. Health status of caregivers of children with cerebral palsy. Child Care Health Dev. 2010;36(5):696-702.

7. Lim JW, Zebrack B. Caring for family members with chronic physical illness: a critical review of caregiver literature. Health Qual Life Outcomes. 2004;2:50

8. Terra VC, Cysneiros RM, Schwartzman JS, Teixeira MC, Arida RM, Cavalheiro EA, et al. Mothers of children with cerebral palsy with or without epilepsy: a quality of life perspective. Disabil Rehabil. 2011;33(5):384-8

9. The World Health Organization Quality of Life assessment (WHOQOL): position paper from the World Health Organization. Soc Sci Med. 1995;41(10):1403-9.

10. Guyatt GH, Naylor CD, Juniper E, Heyland DK, Jaeschke R, Cook DJ. Users' guides to the medical literature. XII. How to use articles about health-related quality of life. Evidence-Based Medicine Working Group. JAMA. 1997;277(15):1232-7.

11. Marquez DX, Bustamante EE, Kozey-Keadle S, Kraemer J, Carrion I. Physical activity and psychosocial and mental health of older caregivers and non-caregivers. Geriatr Nurs. 2012;33(5):358-65.

12. King AC, Baumann K, O'Sullivan P, Wilcox S, Castro C. Effects of moderate-intensity exercise on physiological, behavioral, and emotional responses to family caregiving: a randomized controlled trial. J Gerontol A Biol Sci Med Sci. 2002;57(1):M26-36.

13. Hill K, Smith R, Fearn M, Rydberg M, Oliphant R. Physical and psychological outcomes of a supported physical activity program for older carers. J Aging Phys Act. 2007;15(3):257-71.

14. Hirano A, Suzuki Y, Kuzuya M, Onishi J, Ban N, Umegaki H. Influence of regular exercise on subjective sense of burden and physical symptoms in community-dwelling caregivers of dementia patients: a randomized controlled trial. Arch Gerontol Geriatr. 2011;53(2):e158-63.
15. Takai M, Takahashi M, Iwamitsu Y, Ando N, Okazaki S, Nakajima K, et al. The experience of burnout among home caregivers of patients with dementia: relations to depression and quality of life. Arch Gerontol Geriatr. 2009;49(1):e1-5.

16. Vicente MM, Delgado MG, Fuertes NG, Prieto PP. Effects of an individual home-based physical exercise program in caregivers of Alzheimer patients: a pilot study. RPD. 2009;18(2):255-70.

17. Gillison FB, Skevington SM, Sato A, Standage M, Evangelidou S. The effects of exercise interventions on quality of life in clinical and healthy populations; a meta-analysis. Soc Sci Med. 2009;68(9):1700-10

18. Schuch FB, Vasconcelos-Moreno MP, Fleck M.P. The impact of exercise on Quality of Life within exercise and depression trials: a systematic review. Mental Health Phys Act. 2011;4(2):43-8.

19. Sillanpää E, Häkkinen K, Holviala J, Häkkinen A. Combined strength and endurance training improves health-related quality of life in healthy middle-aged and older adults. Int J Sports Med. 2012;33(12):981-6.

20. Singh NA, Clements KM, Fiatarone MA. A randomized controlled trial of progressive resistance training in depressed elders. J Gerontol A Biol Sci Med Sci. 1997;52(1):M27-35.

21. American College of Sports Medicine. American College of Sports Medicine position stand. Progression models in resistance training for healthy adults. Med Sci Sports Exerc. 2009;41(3):687-708.

22. Ciconelli RM, Ferraz MB, Santos W, Meinão I, Quaresma MR. Tradução para a língua portuguesa e validação do questionário genérico de avaliação de qualidade de vida SF-36 (Brasil SF-36). Rev Bras Reumatol. 1999;36(3):143-50.

23. Gorenstein C, Andrade L. Validation of a Portuguese version of the Beck Depression Inventory and the State-Trait Anxiety Inventory in Brazilian subjects. Braz J Med Biol Res. 1996;29(4):453-7.

24. Medeiros MMC, Feraz BF, Quaresma MR, Menezes AP. Adaptação ao contexto cultural brasileiro e validação do "caregiver burden scale". Rev Bras Reumatol.1998;38(4):193-9.

25. Chiesa LC. Musculação aplicações práticas: técnicas de uso das formas e métodos de treinamento. Rio de Janeiro: Fontoura; 2002.

26. Stroud NM, Minahan CL. The impact of regular physical activity on fatigue, depression and quality of life in persons with multiple sclerosis. Health Qual Life Outcomes. 2009:7:68.

27. Matsudo SM, Matsudo VKR, Neto TLB. Efeitos benéficos da atividade física na aptidão física e saúde mental durante o processo de envelhecimento. Rev Bras Ativ Fís Saúde. 2000;5(2):60-76.

28. Coyle SB. Health-related quality of life of mothers: a review of the esearch. Health Care Women Int. 2009;30(6):484-506.

29. Etkin CD, Farran CJ, Barnes LL, Shah RC. Recruitment and enrollment of aregivers for a lifestyle physical activity clinical trial. Res Nurs Health. 2012;35(1):70-81. 